Check for updates

Cite this: RSC Adv., 2017, 7, 37915

\title{
Precise control of the size of zeolite B-ZSM-5 based on seed surface crystallization $\uparrow$
}

\author{
Chengyi Dai, $\stackrel{+}{a}^{\mathrm{a}}$ Junjie Li, $\stackrel{+}{\mathrm{t}}^{\mathrm{a}}$ Anfeng Zhang, ${ }^{\mathrm{a}}$ Changhong Nie, ${ }^{\mathrm{a}}$ Chunshan Song ${ }^{\mathrm{ab}}$ \\ and Xinwen Guo (D) *a
}

A series of boron-containing ZSM-5 (B-ZSM-5) catalysts with particle sizes from $\sim 153 \mathrm{~nm}$ to $\sim 14.2 \mu \mathrm{m}$ are synthesized by regulating the addition of silicalite-1 seeds. The B-ZSM- 5 particle size with seed addition $(D)$, the seed addition amount $(x)$, the seed particle size $(d)$ and the B-ZSM-5 particle size without seed addition $\left(D_{0}\right)$ are related with by the new function $D^{3}=d^{3} D_{0}^{3} /\left[x D_{0}^{3}+(1-x) d^{3}\right]$, and the particle size of B-ZSM- 5 has been precisely controlled and predicted in both micro (TPABr as template) and nano (TPAOH as template) synthesis systems. The function also shows the evolution of B-ZSM-5 particle size versus the seed amount and size, which helps guide the synthetic choice of the seed amount used or decreasing the seed size to decrease the B-ZSM-5 size for a specific synthesis system. Furthermore, the effect of particle size on the catalytic performance of B-ZSM-5 for the methanol to propylene (MTP) reaction is also investigated. The addition of $1 \mathrm{wt} \% \sim 74 \mathrm{~nm}$ seeds to the synthesis system improves the resulting catalyst lifetime from $46 \mathrm{~h}$ to $794 \mathrm{~h}$.

Received 10th December 2016 Accepted 25th July 2017

DOI: $10.1039 / \mathrm{c} 6 \mathrm{ra} 28030 \mathrm{~g}$

rsc.li/rsc-advances

\section{Introduction}

ZSM-5-type zeolites offer tunable acidity, shape selectivity and hydrothermal stability, leading to their wide use in catalytic operations, ${ }^{\mathbf{1 - 4}}$ such as alkylation, ${ }^{5}$ methane oxidation, ${ }^{6}$ methane aromatization, ${ }^{7}$ environmental catalysis, ${ }^{8-11}$ Fischer-Tropsch synthesis $^{\mathbf{1 2}}$ and methanol to hydrocarbon (MTP, ${ }^{13-15}$ MTO, ${ }^{16}$ $\mathrm{MTA}^{\mathbf{1 7}}$ etc.) reactions. However, the narrow channels of the zeolite inhibit the diffusion of reactants and products, reducing the activity of the catalyst. ${ }^{18}$ On the other hand, the prolonged residence time of the reactants and products in the pores promote side reactions, leading to the decrease of the catalyst stability. ${ }^{19}$ Shortening the length of the zeolite channels is a method to extend the lifetime of the catalyst..$^{20-26}$ The size of ZSM-5 has a significant influence on the activity and stability of the catalyst. ${ }^{27-32}$

Clear solution crystallization and hard/soft template approaches are the two widely adopted methods for the synthesis of ZSM-5 zeolites. $^{33,34}$ However, limited by the

\footnotetext{
${ }^{a}$ State Key Laboratory of Fine Chemicals, PSU-DUT Joint Center for Energy Research, School of Chemical Engineering, Dalian University of Technology, Dalian 116024, P. R. China. E-mail: guoxw@dlut.edu.cn; Fax: +86-411-84986134; Tel: +86-41184986133

${ }^{b}$ EMS Energy Institute, PSU-DUT Joint Center for Energy Research and Department of Energy \& Mineral Engineering, Pennsylvania State University, University Park, Pennsylvania 16802, USA

$\dagger$ Electronic supplementary information (ESI) available: $\mathrm{N}_{2}$ adsorption and desorption isotherms, XRD patterns, $\mathrm{NH}_{3}$-TPD profiles, variations of product selectivity in the MTP reaction. See DOI: $10.1039 / \mathrm{c} 6 \mathrm{ra} 28030 \mathrm{~g}$

\$ C. Dai and J. Li contributed equally.
}

synthesis conditions, the zeolite particle size can only be adjusted within a small range. Seed-induction synthesis, in which the crystal sizes could be well controlled by the addition of seeds, is another well-known method to adjust the particle size of ZSM-5..$^{35,36}$ On the other hand, the introduction of SDA can affect some other properties of zeolites such distribution of aluminium, ${ }^{37}$ the size of zeolite particles ${ }^{38}$ and the ratio between framework and non-framework aluminium, ${ }^{39}$ which are relevant for their catalytic characteristics. Based on seed surface crystallization mechanism, N. Ren et al. proposed a method for facile, rapid, controllable and low-cost synthesis of ZSM-5 zeolite with submicron-size. ${ }^{\mathbf{4 0 - 4 3}}$ Furthermore, crystal growth kinetics, crystalline yield, and physicochemical properties of zeolite nanoparticles as a function of the seed concentration and synthesis temperature were addressed by V. Valtchev et al. ${ }^{44}$ However, most reports focused on the study of the seed role under a single system. Few studies have revealed the effect of the synthesis system on the particle size of ZSM-5 in the presence of seed crystals. $^{38,39}$

In this work, a seed-induction approach has been developed for the micro (TPABr as template) and nano (TPAOH as template) boron-containing ZSM-5 (B-ZSM-5) synthesis systems. We establish a unified relationship to precise control and predict the B-ZSM-5 size given a set amount and size of the seed, which applied to both micro and nano synthesis systems. Furthermore, the performance of B-ZSM-5 was related to its size in the catalytic conversion of methanol to propylene. 


\section{Experimental}

\subsection{Materials}

The reagents used for the preparation of B-ZSM-5 zeolite are tetraethyl orthosilicate (TEOS, analytical grade, Xilong Chemical Co., China), silicon oxide, $30 \mathrm{wt} \%$ in $\mathrm{H}_{2} \mathrm{O}$ colloidal dispersion ( $\mathrm{SiO}_{2}$, Haiyang Chemical Co., China), tetrapropylammonium hydroxide solution, $25 \mathrm{wt} \%$ aqueous solution (TPAOH, Cairui Chemical Co., China), tetrapropylammonium bromide (TPABr, analytical grade, Letai Chemical Co., China), aluminum isopropoxide (analytical grade, Tianjin Institute of Chemical Reagents, China), boric acid (analytical grade, Bodi Chemical Co., China), and ethylamine, 65 wt\% aqueous solution (EA, Tianjin Guangfu Institute of Fine Chemicals Reagents, China).

\subsection{Synthesis of seeds (silicalite-1 nano crystals) with different sizes}

Seeds with various particle sizes were synthesized with the clear solution method. Typically, $50 \mathrm{~g}$ of TEOS was mixed with $70 \mathrm{~g}$ of TPAOH solution and $30 \mathrm{~g}$ of $\mathrm{H}_{2} \mathrm{O}$. The molar composition of the synthesis mixture was 1TEOS : 0.36TPAOH : $19 \mathrm{H}_{2} \mathrm{O}$. After being stirred for $12 \mathrm{~h}$ at $35{ }^{\circ} \mathrm{C}$, the gel was transferred into a $200 \mathrm{~mL}$ Teflon-lined steel autoclave and crystallized for 3 days; the crystallization temperature was 80,100 and $120^{\circ} \mathrm{C}$, respectively. The function of average seed size $(d) v s$. crystallization temperature $(T)$ is eqn (1), as shown in Fig. 2. The obtained gel solution was directly used as the seed without any centrifugation or washing process.

\subsection{Synthesis of B-ZSM-5 in micro (TPABr as template) and nano (TPAOH as template) synthesis systems}

In the micro synthesis system, colloidal silica, boric acid and aluminum isopropoxide were used as silicon, boron and aluminum sources, respectively. TPABr was used as the template and aqueous EA as the base. Molar composition of the gel was $1 \mathrm{SiO}_{2}: 0.15 \mathrm{TPABr}: 0.4 \mathrm{~B}_{2} \mathrm{O}_{3}: 0.0025 \mathrm{Al}_{2} \mathrm{O}_{3}: 1 \mathrm{EA}: 17 \mathrm{H}_{2} \mathrm{O}$. Typically, TPABr was dissolved with silica solution and afterwards with a solution of EA. The mixture was aged at room temperature for $30 \mathrm{~min}$ under mechanical stirring followed by adding a solution of aluminum isopropoxide and boric acid.

In the nano synthesis system, B-ZSM-5 was obtained from a clear solution with the composition: 1TEOS : $0.27 \mathrm{TPAOH}: 0.4 \mathrm{~B}_{2} \mathrm{O}_{3}: 0.0025 \mathrm{Al}_{2} \mathrm{O}_{3}: 37 \mathrm{H}_{2} \mathrm{O}$. Typically, the mixture of TEOS and TPAOH was stirred at $35^{\circ} \mathrm{C}$ for $3 \mathrm{~h}$ followed by adding a solution of aluminum isopropoxide and boric acid.

After mixing the initial reactants, milky-way colloidal dispersion was obtained in the micro synthesis system, and clear solution was obtained in the nano synthesis system. ${ }^{45}$ The $\mathrm{pH}$ of the reaction mixtures in both synthesis system were $13 \pm$ 0.2 . Then, a certain amount (defined as $x$ ) of seed was added to the mixture. In this work, $m$ represents the total amount (mass) of silica in the seed, $M$ represents the total amount (mass) of silica in the reaction mixture including the mass of silica in the seed, i.e., $x=m / M=m /\left(M_{0}+m\right)$, where $M_{0}=(M-m)$ is the total amount (mass) of silica present in the reaction mixture, before addition of seed. The synthesis was performed at $170{ }^{\circ} \mathrm{C}$ for 3 days. The product was collected by centrifugation and dried overnight at $100{ }^{\circ} \mathrm{C}$. Finally, the template was removed by calcination in static air at $540{ }^{\circ} \mathrm{C}$ for $6 \mathrm{~h}$.

\subsection{Characterization}

Powder X-ray diffraction (XRD) patterns were recorded on a Rigaku Smart Lab diffractometer, using a nickel-filtered CuKa $\mathrm{X}$-ray source at a scanning rate of 0.02 over the range between $5^{\circ}$ and $50^{\circ}$. Scanning electron microscopy (SEM) images were obtained on a Hitachi S-5500 instrument with acceleration voltages of $3 \mathrm{kV}$ and $5 \mathrm{kV}$. Some samples were sputtered with a thin film of gold. The particle size distributions of seed crystals and BZSM-5 products were determined by counting 100 particles in the SEM images. For irregular spheres, we used the arithmetic mean of the long and short sides to represent the diameter. Particles size distribution curves of the products shown in Fig. 6 and 9 were determined with a Zetasizer 1000 laser lightscattering (LLS) particle size analyzer. $\mathrm{N}_{2}$ isotherms at $77 \mathrm{~K}$ were measured in a Quantachrome QUADRASORB SI gas adsorption analyzer. Prior to the measurement, the samples were degassed in vacuum at $300{ }^{\circ} \mathrm{C}$ for $10 \mathrm{~h}$. The BrunauerEmmett-Teller (BET) method was applied to calculate the total surface area, while the $t$-plot method was used to discriminate between micro- and mesoporosity. In the $t$-plot, the reported mesopore surface area $\left(S_{\text {meso }}\right)$ consists of contributions from the outer surface of the particles as well as mesopores and macropores. The elemental analysis of catalysts was conducted on a Perkin Elmer OPTIMA 2000DV ICP Optical Emission Spectrometer. Thermogravimetric analysis (TGA) was performed on an SDT Q600 (TA Instruments, USA) in the temperature range of $25-800{ }^{\circ} \mathrm{C}$ under air at a heating rate of $10{ }^{\circ} \mathrm{C} \mathrm{min}^{-1}$. Ammonia temperature programmed desorption $\left(\mathrm{NH}_{3}\right.$-TPD) of the sample was measured by heating the sample from 100 to $720^{\circ} \mathrm{C}$ at a rate of $10^{\circ} \mathrm{C} \mathrm{min}{ }^{-1}$. Each sample was first treated at $300{ }^{\circ} \mathrm{C}$ in $\mathrm{Ar}$ flow for $1 \mathrm{~h}$, and then cooled to room temperature. The effluent stream was monitored continuously with a thermal conductivity detector (TCD) to determine the rate of ammonia desorption.

\subsection{Catalytic tests}

The MTP reaction was performed at $500{ }^{\circ} \mathrm{C}$ in a fixed-bed reactor under atmospheric pressure. The catalyst loading was $1.0 \mathrm{~g}$ (10$20 \mathrm{mesh}$ ) and the WHSV for methanol was $1.0 \mathrm{~h}^{-1}$ with a $\mathrm{MeOH} /$ $\mathrm{H}_{2} \mathrm{O}$ molar ratio of $1: 5$. The gas products were analyzed by a gas chromatograph equipped with a Flame Ionization Detector (FID) and a HP-PLOTQ capillary column $30 \mathrm{~m}$ long. The liquid products were analyzed by a gas chromatograph equipped with a FID and an INNOWAX capillary column $60 \mathrm{~m}$ long.

\section{Results and discussions}

\subsection{Effect of the seed size in micro-B-ZSM-5 synthesis system}

Initially, experiments were performed to study the effect of seed sizes on B-ZSM-5 formation. Silicalite-1 zeolites with different crystal sizes were used as seeds. Fig. 1 displays SEM images of silicalite- 1 seeds crystallized at 80,100 , and $120^{\circ} \mathrm{C}$, respectively. 

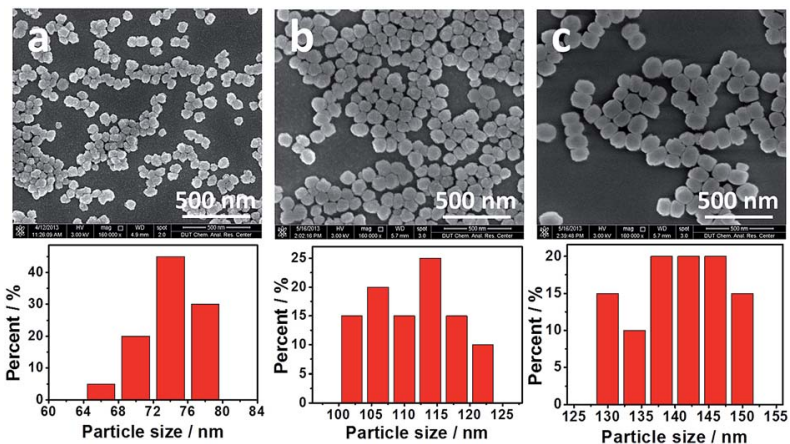

Fig. 1 SEM images of silicalite-1 seeds prepared at different temperatures, and particle size distributions of them, (a) $80^{\circ} \mathrm{C}$, (b) $100{ }^{\circ} \mathrm{C}$, (c) $120^{\circ} \mathrm{C}$.

Based on the results of the measurement of 100 randomly distributed particles by SEM observations, the particle size distributions were derived. The average sizes of the seeds are affected by the crystallization temperature, with seed size finely adjusted to 74,111 , and $141 \mathrm{~nm}$. The function of average seed size $(d) v s$. crystallization temperature $(T)$ is eqn (1), as shown in Fig. 2.

$$
d=1.7 T-59
$$

The XRD patterns show that the seeds are MFI zeolite with good crystallinity (Fig. S1†).

The B-ZSM-5 zeolite synthesized in the absence of seed had large crystals of $14.2 \mu \mathrm{m}$ in diameter (Fig. 3a and S2a $\dagger$ ). When 1 $\mathrm{wt} \%$ seeds were added in the synthesis system, the crystal sizes of the samples decreased to sub-micrometer and could be well tuned by adopting seeds with different sizes (Fig. 3b-d). With 74, 111 and $141 \mathrm{~nm}$ seed sizes, the average particle sizes of BZSM-5 are 336, 536 and $691 \mathrm{~nm}$, respectively (Fig. S2b-d $\dagger$ ). Fig. 4 shows that the relationship between the sizes of the B-

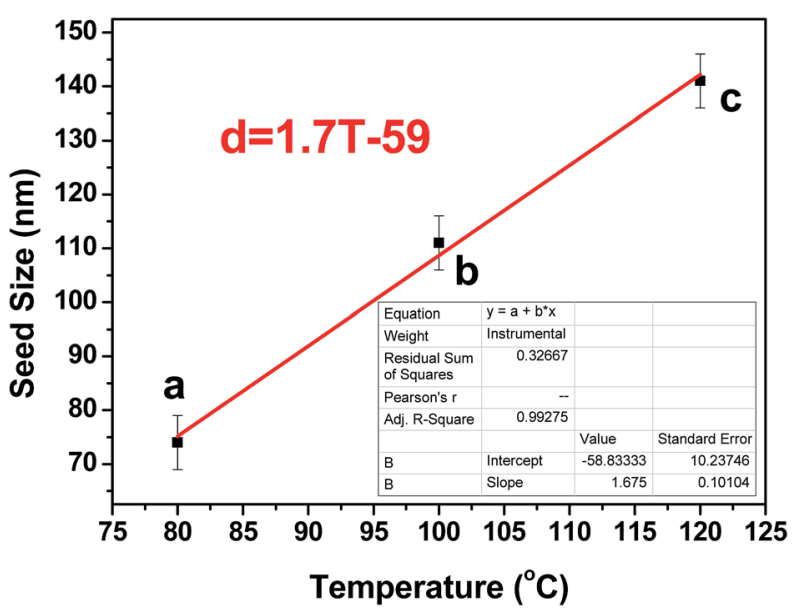

Fig. 2 Relationship between the crystallization temperature and particle sizes of silicalite- 1 seeds, (a) $80^{\circ} \mathrm{C}, 74 \mathrm{~nm}$, (b) $100{ }^{\circ} \mathrm{C}, 111 \mathrm{~nm}$, (c) $120^{\circ} \mathrm{C}, 141 \mathrm{~nm}$.
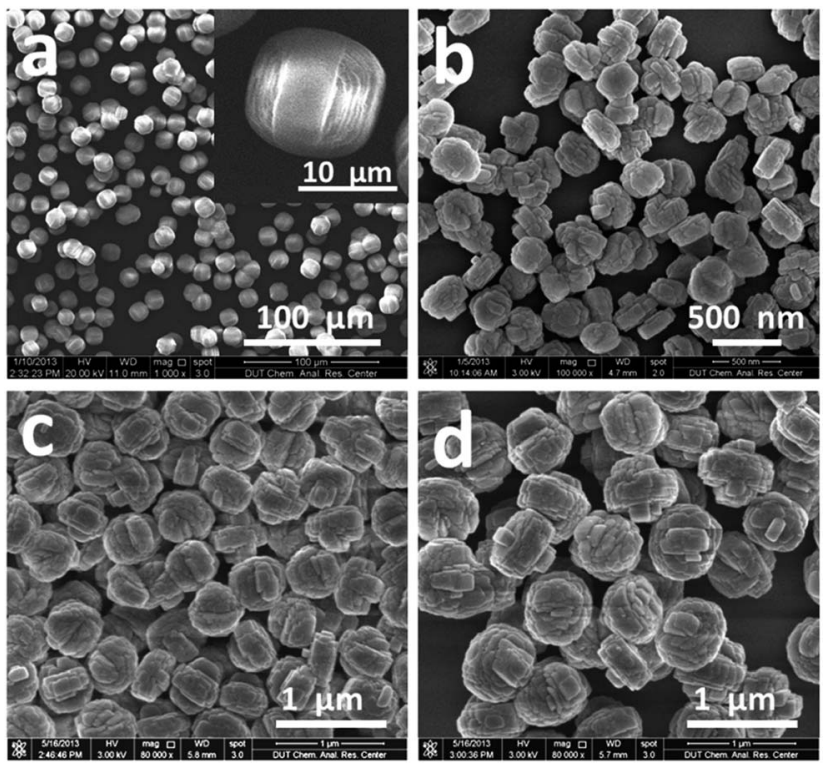

Fig. 3 SEM images of the B-ZSM-5 nanoparticles with different sizes, (a) B-ZSM-5 zeolite synthesized in the absence of seed, (b-d) the amount of seed addition was $1 \%$ and the seed size was 74,111 and $141 \mathrm{~nm}$, respectively.

ZSM-5 zeolite and seed is linear. The function of average BZSM-5 size $(D)$ vs. seed size $(d)$ is eqn (2).

$$
D=5.3 d-55
$$

The XRD patterns show that all the B-ZSM-5 samples are MFI zeolite with good crystallinity (Fig. S3 $\dagger$ ). Fig. S4† shows $\mathrm{N}_{2}$ adsorption/desorption isotherms of the B-ZSM- 5 crystals grown from seed-free gels and gels containing $1 \mathrm{wt} \%$ silicalite- 1 seeds with different sizes. With the particle size decreases, the adsorption isotherms change from type I to type II because of the increased intercrystal pores, while the external surface area

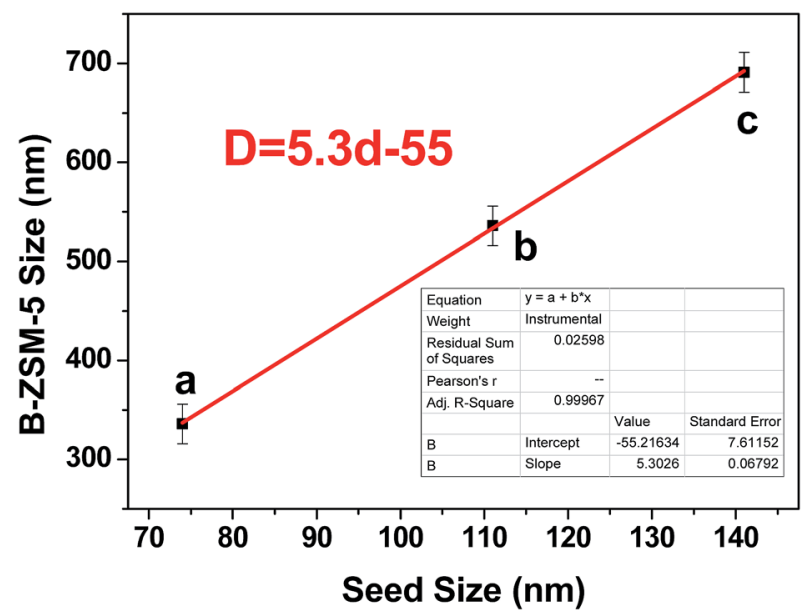

Fig. 4 Relationship between the sizes of the B-ZSM-5 zeolite and seed sizes, (a) $336 \mathrm{~nm}$, (b) $536 \mathrm{~nm}$, (c) $691 \mathrm{~nm}$. The amount of seed addition was $1 \%$ and the seed size was 74,111 and $141 \mathrm{~nm}$, respectively. 
increases from 24 to $44 \mathrm{~m}^{2} \mathrm{~g}^{-1}$, and the pore volume increases from 0.17 to $0.29 \mathrm{~cm}^{3} \mathrm{~g}^{-1}$ (Table S1†).

\subsection{Effect of the amount of seeds in micro-B-ZSM-5 synthesis system}

It is well known that the size of final crystalline products in the seed-induction synthesis depends on both amount and size of seed addition..$^{40}$ To check the influence of the amount of seed, $0.1-10 \mathrm{wt} \%$ of $74 \mathrm{~nm}$ silicalite-1 seed crystals were added into the reaction mixture. The average size of formed B-ZSM-5 particles was 153, 195, 336 and $676 \mathrm{~nm}$ when the amount of seed addition was 10, 5, 1 and $0.1 \%$, respectively (Fig. 5 and $\mathrm{S} 5 \dagger$ ). In order to ensure the accuracy and reliability of particle size statistics from SEM images, the dynamic light scattering (DLS) was also used to measure the particle size distribution of B-ZSM-5 nanoparticles, as shown in Fig. 6. The average particle size of B-ZSM-5 was 223, 316 and $672 \mathrm{~nm}$ when the amount of seed addition was 5,1 and $0.1 \%$, respectively, which was similar with the statistical results from SEM images.

By using experimental data of the amount of silicalite- 1 seed and B-ZSM-5 crystal size with the polynomial fitting, a good function of B-ZSM-5 size $(D) v s$. seed amount $(x)$ is obtained, the function is eqn (3), as shown in Fig. 7.

$$
D=74 x^{-0.32}
$$

Thus, the size of the B-ZSM- 5 crystals can be predicted by this correlation. For example, when the amount of seed was set as $0.5 \mathrm{wt} \%(x=0.005)$, the size of the B-ZSM- 5 crystal calculated from the function was $403 \mathrm{~nm}$, while the experiment gave a crystal with a size of $417 \mathrm{~nm}$ (Fig. 5b and S5b†).

Fig. S6 $\uparrow$ shows $\mathrm{N}_{2}$ adsorption/desorption isotherms of the BZSM-5 crystal grown from gels containing $0.1-10 \mathrm{wt} \%$ of $74 \mathrm{~nm}$
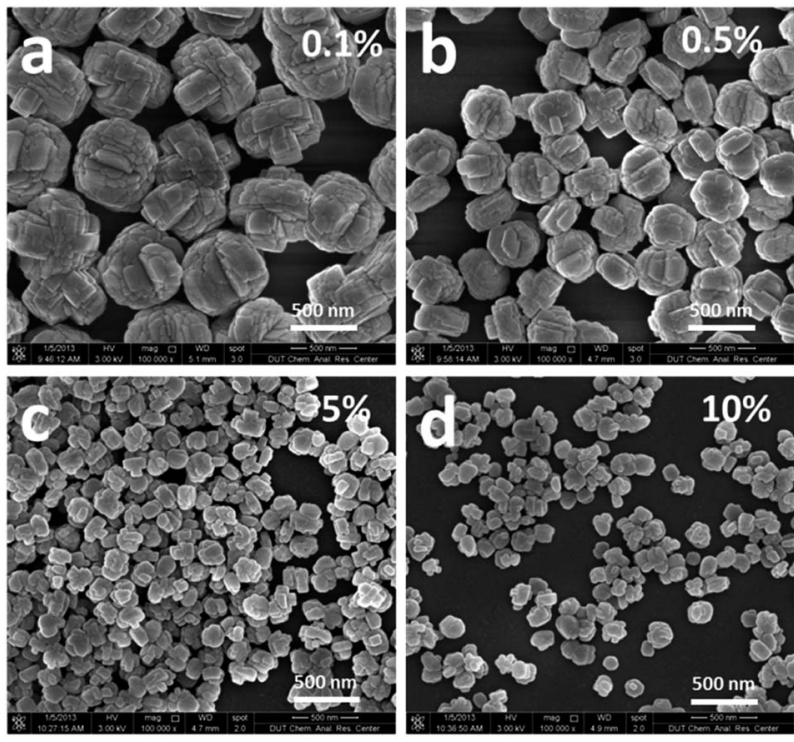

Fig. 5 SEM images of the B-ZSM-5 nanoparticle with different sizes. The seed size was $74 \mathrm{~nm}$ and the amount of seed addition was $0.1,0.5$, 5 and $10 \%$, respectively.

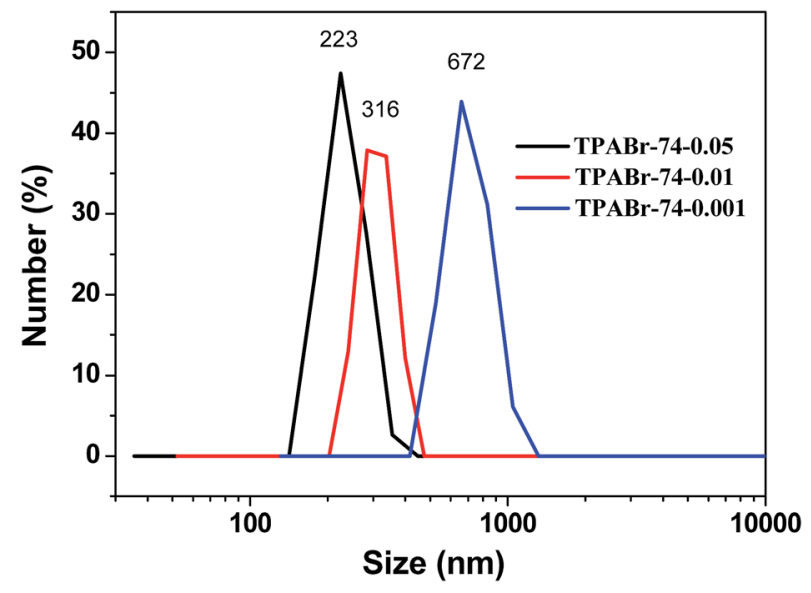

Fig. 6 Particle size distribution by number of the B-ZSM-5 synthesized in the micro synthesis system. The seed size was $74 \mathrm{~nm}$ and the amount of seed addition was 5,1 and $0.1 \%(x=0.05,0.1$ and 0.01$)$, respectively.

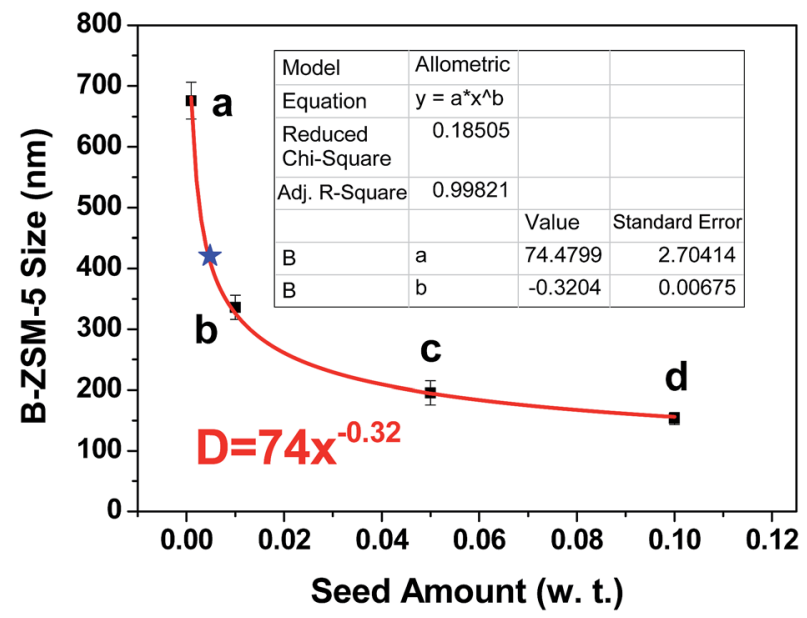

Fig. 7 Relationship between the sizes of the B-ZSM- 5 zeolite and seed addition amount in the micro synthesis system, seed size was $74 \mathrm{~nm}$, (a) $0.001,676 \mathrm{~nm}(x, D)$, (b) $0.01,336 \mathrm{~nm}$, (c) $0.05,195 \mathrm{~nm}$, (d) 0.1 , $153 \mathrm{~nm}$, (blue five-pointed star) $0.005-417 \mathrm{~nm}$.

silicalite-1 seeds. With decreasing particle size, the $\mathrm{N}_{2}$ uptake at high $P / P_{0}$ is enhanced. This is associated with capillary condensation of nitrogen within the intercrystal pores. ${ }^{3}$ The BET surface area and the micropore volume of the sample have almost no change when the size of crystals decreases from 676 to $153 \mathrm{~nm}$, while the external surface area increases from 34 to $47 \mathrm{~m}^{2} \mathrm{~g}^{-1}$, and the pore volume increases from 0.23 to $0.34 \mathrm{~cm}^{3}$ $\mathrm{g}^{-1}$ (Table S2 $\dagger$ ). The XRD patterns show that the crystals are MFI zeolite with good crystallinity (Fig. S7†).

\subsection{Effect of the amount of seeds in nano-B-ZSM-5 synthesis system}

In the nano-B-ZSM-5 synthesis system, $0.1-10 \mathrm{wt} \%$ of $74 \mathrm{~nm}$ silicalite-1 seed crystals was added into the reaction mixture. The SEM image shows that the average B-ZSM-5 crystal size was 
$268 \mathrm{~nm}$ in the absence of seeds (Fig. 8a and S8a $\dagger$ ). The crystal sizes of the samples became slightly smaller with an increasing amount of seeds (Fig. $8 \mathrm{~b}-\mathrm{f}$ and $\mathrm{S} 8 \mathrm{~b}-\mathrm{f} \dagger$ ). With the increase of the amount of seeds from 0.1 to $10 \mathrm{wt} \%$, the average size of B-ZSM-5 was decreased from $266 \mathrm{~nm}$ to $157 \mathrm{~nm}$ (Fig. 10).

\subsection{Comparative studies of the sizes of B-ZSM-5 crystals} synthesized in micro- or nano-synthesis system

Without the addition of seed, there are significant differences between the sizes of B-ZSM-5 crystals synthesized in TPABr medium and TPAOH medium, which are $14.2 \mu \mathrm{m}$ in TPABr medium and $268 \mathrm{~nm}$ in TPAOH medium. This is because fewer nuclei are produced in the TPABr medium. Because the alkalinities of both the systems are the same $(\mathrm{pH}=13$; see Experimental), it can be assumed that content of TPA ${ }^{+}$ions (expressed as the molar ratio $\left.\left[\mathrm{TPA}^{+} / \mathrm{SiO}_{2}\right]\right)$ is the main factor which determines the specific number of nuclei (number of nuclei per unit mass of the reaction mixture) and consequently, the size of the MFI crystals formed in the investigated reaction mixtures, ${ }^{\mathbf{4 6 - 4 9}}$ i.e., higher specific number of nuclei and smaller crystal size $(268 \mathrm{~nm})$ in the nano-system $\left(\left[\mathrm{TPA}^{+} / \mathrm{SiO}_{2}\right]=0.27\right)$ and lower specific number of nuclei and larger crystal size $(14.2 \mathrm{~m})$ in the
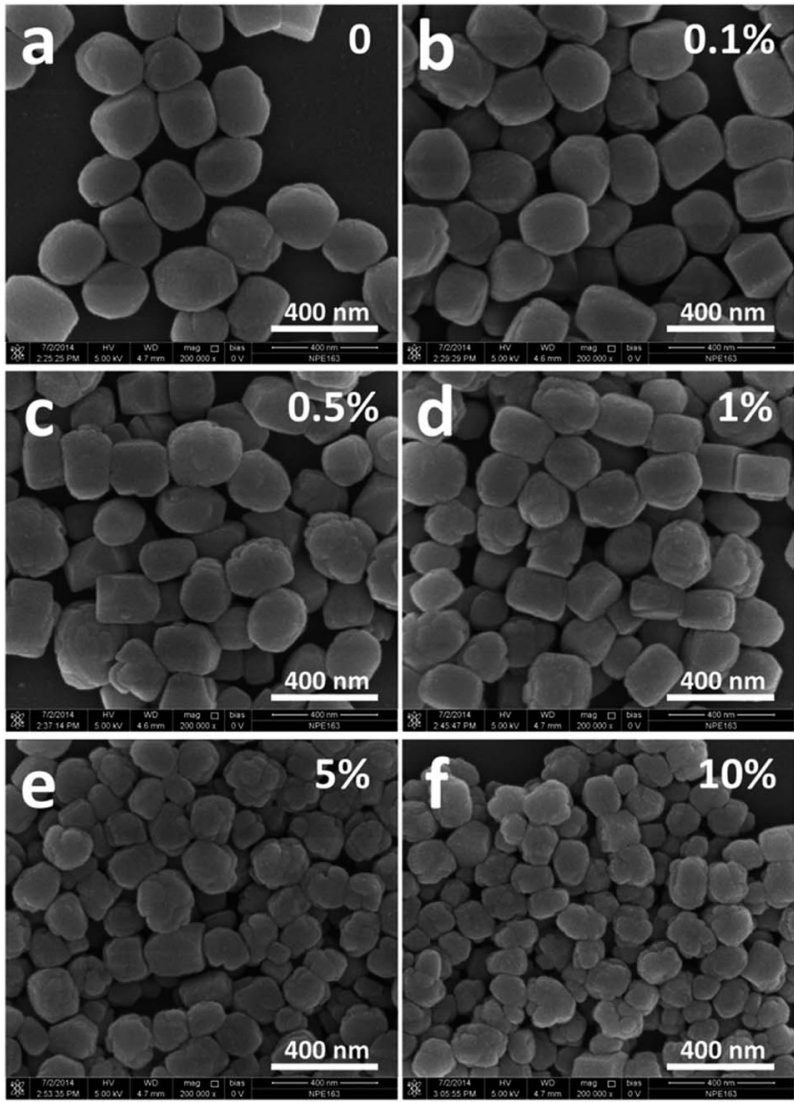

Fig. 8 SEM images of B-ZSM-5 crystals synthesized in TPAOH medium with different particle sizes. (a) $268 \mathrm{~nm}$, (b) $266 \mathrm{~nm}$, (c) $235 \mathrm{~nm}$, (d) $221 \mathrm{~nm}$, (e) $188 \mathrm{~nm}$, (f) $157 \mathrm{~nm}$. The seed size was $74 \mathrm{~nm}$ and the amount of seed addition was 0 (a), 0.1 (b), 0.5 (c), 1 (d), 5 (e) and $10 w t \%(f)$. micro-system $\left(\left[\mathrm{TPA}^{+} / \mathrm{SiO}_{2}\right]=0.15\right)$. However, when the seed was added to the synthesis system, the size of B-ZSM-5 synthesized in the TPABr medium decreased significantly, while it decreased slightly in the TPAOH medium. For example, when $0.1 \mathrm{wt} \%$ seed $(74 \mathrm{~nm})$ was added in the TPABr system, the size of B-ZSM-5 crystal decreased from $14.2 \mu \mathrm{m}$ to $676 \mathrm{~nm}$, but it decrease from $268 \mathrm{~nm}$ to $266 \mathrm{~nm}$ in the TPAOH medium. Furthermore, when the amount of seed $(74 \mathrm{~nm})$ was increased to $5 \mathrm{wt} \%$, the sizes of B-ZSM- 5 crystals synthesized in TPABr and TPAOH media were almost the same, which were 195 and $188 \mathrm{~nm}$, respectively. Fig. 9 shows the particle size distribution of the B-ZSM-5 synthesized in the nano synthesis system with DLS measurement method. The average particle size of B-ZSM-5 was 182, 221 and $280 \mathrm{~nm}$ when the amount of seed addition was 5,1 and $0.1 \%$, respectively, which was similar with the statistical results from SEM images.

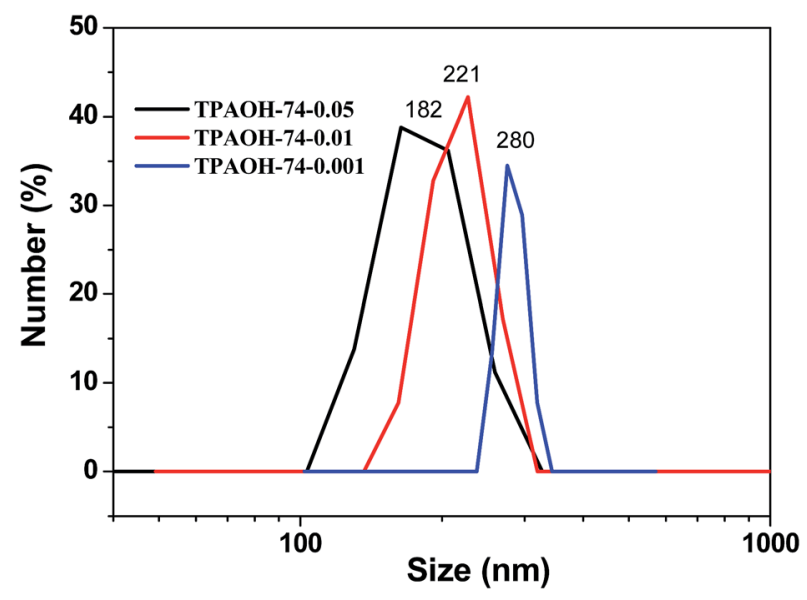

Fig. 9 Particle size distribution by number of the B-ZSM-5 synthesized in the nano synthesis system. The seed size was $74 \mathrm{~nm}$ and the amount of seed addition was 5,1 and $0.1 \%$, respectively.

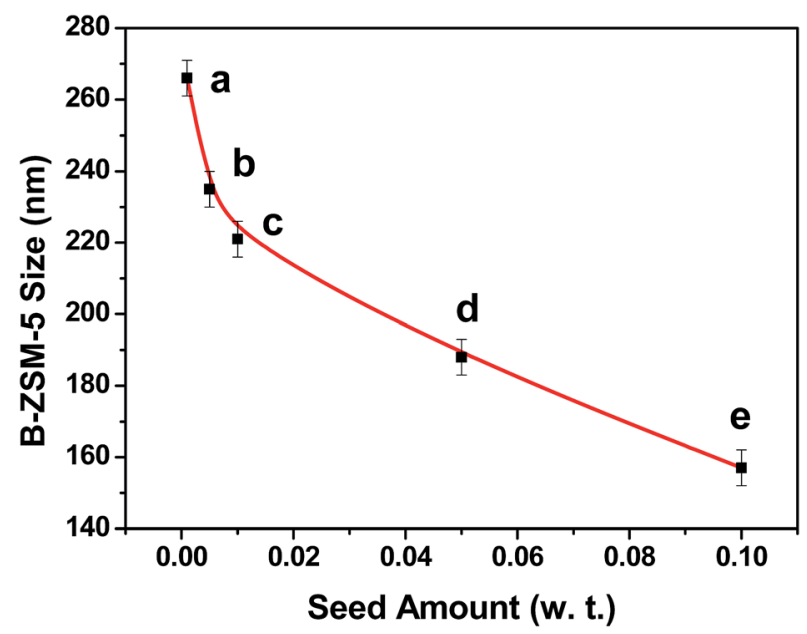

Fig. 10 Relationship between the sizes of the B-ZSM-5 zeolite and seed addition amount in the nano synthesis system, seed size was $74 \mathrm{~nm}$, (a) $0.001,266 \mathrm{~nm}(x, D),(b) 0.005,235 \mathrm{~nm}$, (c) 0.01, $221 \mathrm{~nm}$, (d) $0.05,188 \mathrm{~nm}$, (e) $0.1,157 \mathrm{~nm}$. 
It is well known that the crystallization of B-ZSM-5 zeolites occurs on the surface of nuclei, ${ }^{40}$ and the number of nuclei produced in the synthesis system does not change when the seed is added (Scheme 1). Then, the number of nuclei in the system is equal to the number generated and the added seed crystals (eqn (6)). In the equation, $m$ is the mass of silicalite-1 seed; $M$ is the mass of B-ZSM-5 product; $x$ represents $m / M$ (eqn (4)); $\rho$ is the density of MFI zeolite; $D, d$, and $D_{0}$ are the diameter of the B-ZSM-5 particles with seed addition, the seed itself, and the B-ZSM-5 particle without seed addition, respectively. $6 m / \pi d^{3} \rho$ represents the number of silicalite- 1 seed crystals, and $6(M-m) / \pi D_{0}{ }^{3} \rho$ represents the number of generated nuclei. We arrive at eqn (7) by substituting eqn (6) into eqn (5). With eqn (7), the final crystal size of micro- or nano-synthesis systems with different seed sizes and amount can be easily calculated (Table 1). More interestingly, using the calculated data, we can get the relationship between the sizes of B-ZSM-5 zeolite and the seed size or amount in different synthesis systems (Fig. 11, we choose different synthesis system which $D_{0}$ is $14.2 \mu \mathrm{m}, 1 \mu \mathrm{m}, 500 \mathrm{~nm}$ and $300 \mathrm{~nm})$. The calculated curves show that the sizes of B-ZSM-5 crystals of different synthesis systems are almost the same when the amount of seed $(74 \mathrm{~nm})$ is larger than $3 \mathrm{wt} \%$. In the micro-synthesis system (e.g. $D_{0}$ is

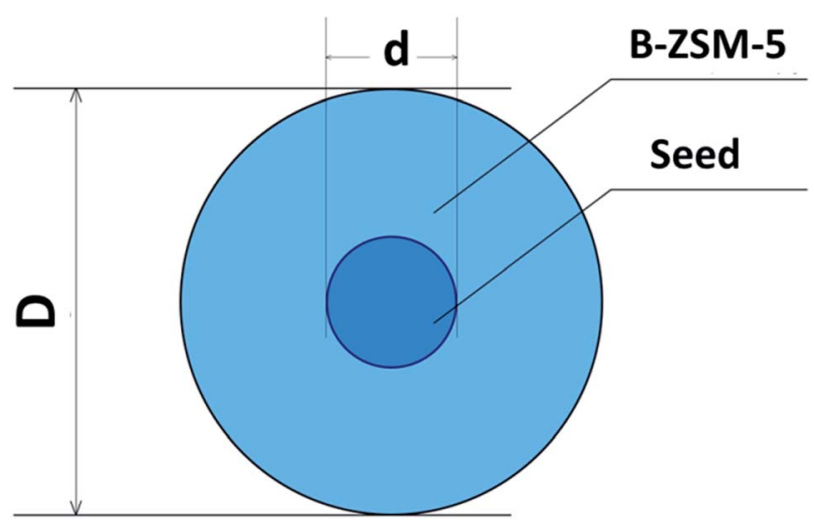

Scheme 1 Schematically illustration of the seed surface crystallization.

Table 1 The comparison of final crystal size of synthesized B-ZSM-5 zeolites from calculation and SEM determination

\begin{tabular}{llllll}
\hline$d[\mathrm{~nm}]$ & $D_{0}[\mathrm{~nm}]$ & $x[\mathrm{wt}]$ & Template & $D^{a}[\mathrm{~nm}]$ & $D^{b}[\mathrm{~nm}]$ \\
\hline 141 & 14200 & 0.01 & TPABr & 654 & 691 \\
111 & 14200 & 0.01 & TPABr & 515 & 536 \\
74 & 14200 & 0.01 & TPABr & 343 & 336 \\
74 & 14200 & 0.001 & TPABr & 740 & 676 \\
74 & 14200 & 0.005 & TPABr & 433 & 417 \\
74 & 14200 & 0.05 & TPABr & 201 & 195 \\
74 & 14200 & 0.1 & TPABr & 159 & 153 \\
74 & 268 & 0.001 & TPAOH & 264 & 266 \\
74 & 268 & 0.005 & TPAOH & 250 & 235 \\
74 & 268 & 0.01 & TPAOH & 236 & 221 \\
74 & 268 & 0.05 & TPAOH & 180 & 188 \\
74 & 268 & 0.1 & TPAOH & 150 & 157
\end{tabular}

${ }^{a}$ Calculated from eqn (4). ${ }^{b}$ Determined from the SEM images.
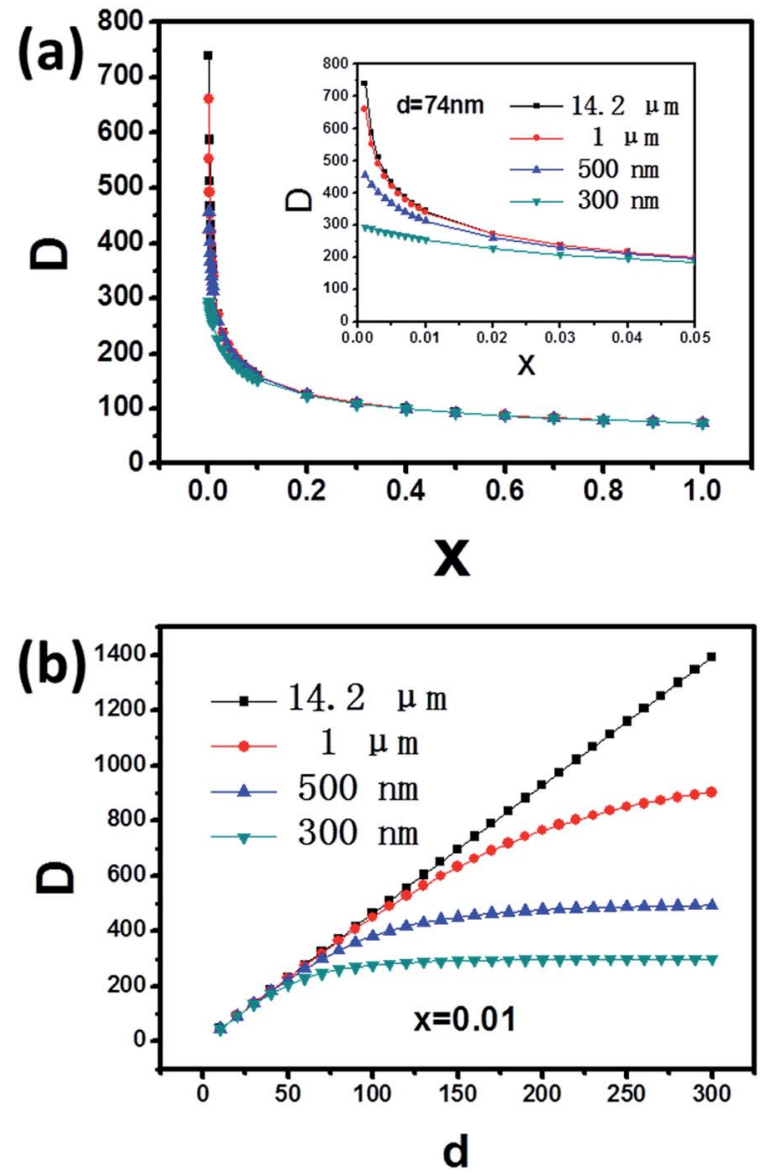

Fig. 11 (a) Relationship between the sizes of B-ZSM-5 zeolite and the seed addition amount in different synthesis systems $\left(D_{0}=14.2 \mu \mathrm{m}, 1\right.$ $\mu \mathrm{m}, 500 \mathrm{~nm}$ and $300 \mathrm{~nm}$, respectively), the seed size was $74 \mathrm{~nm}$. (b) Relationship between the sizes of B-ZSM- 5 zeolite and the seed size in different synthesis systems ( $D_{0}=14.2 \mu \mathrm{m}, 1 \mu \mathrm{m}, 500 \mathrm{~nm}$ and $300 \mathrm{~nm}$, respectively), the seed addition amount was $1 \mathrm{wt} \%$. The data in curves are calculated from eqn (4).

$14.2 \mu \mathrm{m})$, when the size of seed is smaller than $300 \mathrm{~nm}$, eqn (7) can be simplified into eqn (8), which agree with the relationships shown in Fig. 7 (eqn (3)).

$$
\begin{gathered}
x=m / M \\
\pi D^{3} \rho / 6=M / n \\
n=6 m / \pi d^{3} \rho+6(M-m) / \pi D_{0}^{3} \rho \\
=6\left(x M D_{0}^{3}+M d^{3}-x M d^{3}\right) / \pi d^{3} D_{0}{ }^{3} \rho \\
D^{3}=d^{3} D_{0}{ }^{3} /\left[x D_{0}{ }^{3}+(1-x) d^{3}\right] \\
D=d x^{-1 / 3}
\end{gathered}
$$

\subsection{Effect of particle sizes of B-ZSM-5 on the MTP reaction}

Fig. S9† shows $\mathrm{NH}_{3}$-TPD profiles of B-ZSM-5 with different particle sizes synthesized in the micro-synthesis system by using $0,0.1$ and $1 \% 74 \mathrm{~nm}$ seed, respectively. The desorption 
peak at about $200{ }^{\circ} \mathrm{C}$ could be assigned to weakly adsorbed ammonia on B-ZSM-5, and the peak at about $380{ }^{\circ} \mathrm{C}$ could be ascribed to strong acid sites of B-ZSM-5. From Fig. S9 and Table $\mathrm{S} 3, \dagger$ it can be seen that the acidity and chemical compositions were similar among the B-ZSM-5 samples with different particle sizes. Meanwhile, both the external surface area and total pore volume show quasi-linear dependence with the particle size of B-ZSM-5, gradually increasing upon decreasing the size of the particle (Fig. 12). These properties prove more beneficial for the transport of reactants and products, while increasing the carbon capacity.

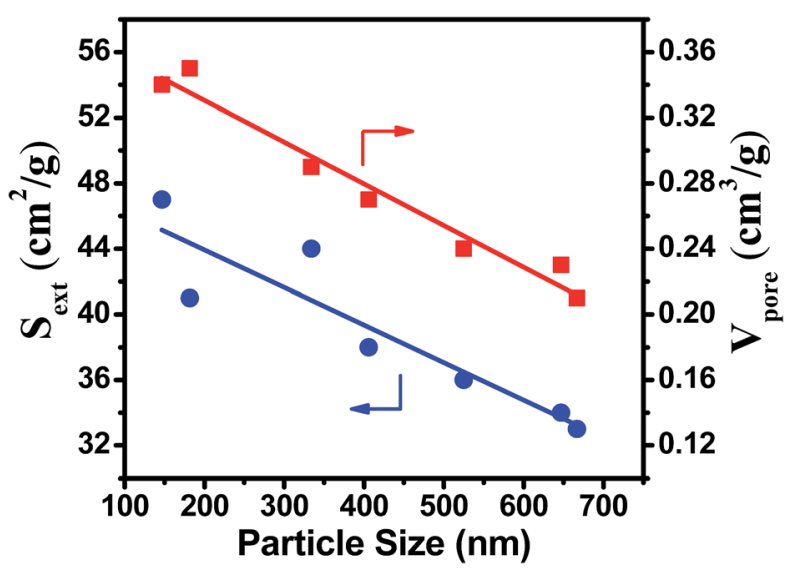

Fig. 12 Influence of particle size on external surface area and total pore volume of B-ZSM-5 zeolite.

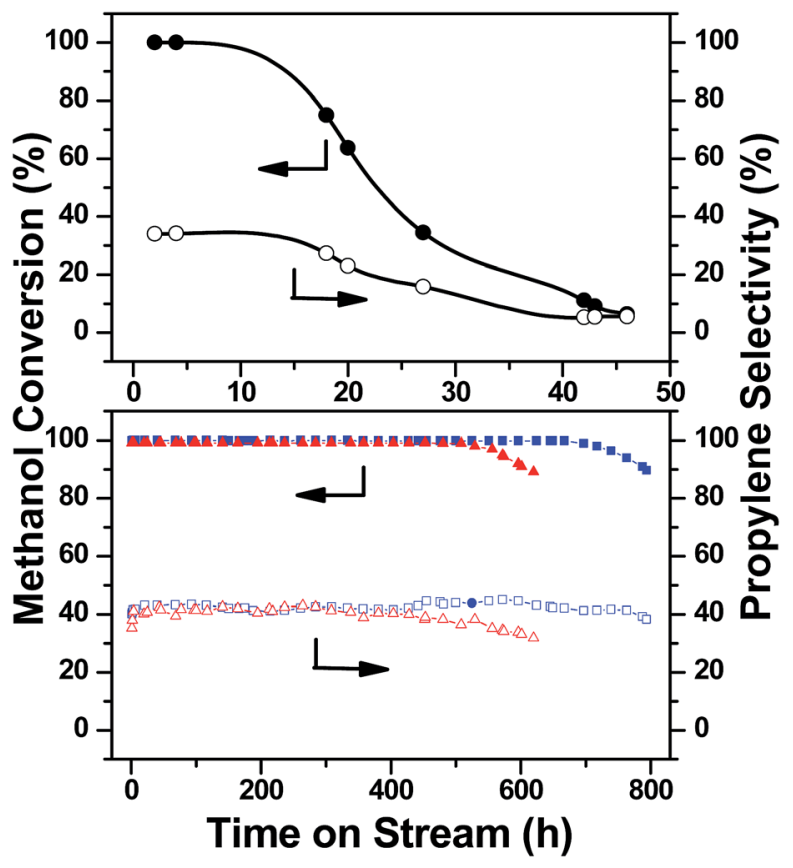

Fig. 13 Variations of methanol conversion and propylene selectivity over $14.2 \mu \mathrm{m}$ (black circle), $676 \mathrm{~nm}$ (red triangle) and $336 \mathrm{~nm}$ (blue square) B-ZSM-5 as a function of the time on stream. Reaction conditions: WHSV $=1 \mathrm{~h}^{-1}, T=500^{\circ} \mathrm{C}, n\left(\mathrm{CH}_{3} \mathrm{OH}\right): n\left(\mathrm{H}_{2} \mathrm{O}\right)=1: 5, P_{\text {total }}$ $=1 \mathrm{~atm}$.
Table 2 Catalyst lifetime of ZSM-5 for MTP reaction in the present and previous works

\begin{tabular}{llllll}
\hline $\begin{array}{l}\text { WHSV } \\
{\left[\mathrm{h}^{-1}\right]}\end{array}$ & $T\left[{ }^{\circ} \mathrm{C}\right]$ & $\begin{array}{l}n\left(\mathrm{CH}_{3} \mathrm{OH}\right): \\
n\left(\mathrm{H}_{2} \mathrm{O}\right)\end{array}$ & $\begin{array}{l}P_{\text {total }} \\
{[\mathrm{atm}]}\end{array}$ & $\begin{array}{l}\text { Cat. lifetime }^{a} \\
{[\mathrm{~h}]}\end{array}$ & Ref. \\
\hline 1 & 500 & $1: 5$ & 1 & 794 & This work \\
1.25 & 460 & $1: 5$ & 1 & 375 & 13 \\
1.8 & 460 & $1: 3$ & 1 & 700 & 14 \\
0.75 & 460 & $1: 5$ & 1 & 50 & 15 \\
1 & 460 & $1: 1.8$ & 1 & $<60$ & 28
\end{tabular}

${ }^{a}$ Methanol conversion is greater than $90 \%$.

Fig. 13 and $\mathrm{S} 10 \uparrow$ illustrate the methanol conversion and the products selectivity for the B-ZSM-5 catalysts with sizes $14.2 \mu \mathrm{m}$, $676 \mathrm{~nm}$ and $336 \mathrm{~nm}$, respectively. During the first 10 hours, the conversions of the three catalysts were all higher than $99 \%$, and the selectivity were $34 \%, 40 \%$ and $40 \%$, respectively. $10 \mathrm{~h}$ later, the methanol conversion and selectivity of propylene dropped for the B-ZSM-5 with a large particle size of $14.2 \mu \mathrm{m}$, while the catalytic performances of B-ZSM-5 with 676 and $336 \mathrm{~nm}$ sizes can be maintained stable for more than 600 hours $(620 \mathrm{~h}$ and $794 \mathrm{~h}$, respectively). As expected, the shorter channels and higher pore volume improved catalyst performance for the MTP reaction.

The coke content in the deactivated catalysts was determined using a detailed thermal analysis. Fig. S11† shows the mass loss of the deactivated catalysts during coke combustion. The catalyst with $14.2 \mu \mathrm{m}$ particle size has a coke content of around $9.8 \%$; whereas the $336 \mathrm{~nm}$ size catalyst showed a mass decrease of $23 \%$ after combusting all coke deposits. To compare the coke formation rate, we also determined the mass loss of the $336 \mathrm{~nm}$ catalyst after 46 hours reaction, and the coke content is $6.4 \%$, which is smaller than that of $14.2 \mu \mathrm{m}$ catalyst. These results identify that the catalyst with smaller particle size can indeed limit carbon formation while also having a larger carbon capacity (Table 2 ).

\section{Conclusions}

A series of B-ZSM-5 zeolites with particle sizes from $\sim 153 \mathrm{~nm}$ to $\sim 14.2 \mu \mathrm{m}$ were synthesized by regulating the addition of silicalite-1 seed, and we established a unified function $D^{3}=$ $d^{3} D_{0}{ }^{3} /\left[x D_{0}{ }^{3}+(1-x) d^{3}\right]$ to precise control or predict the B-ZSM-5 size give the seed amount and size. The model shows that the sizes of B-ZSM-5 crystals synthesized in different template systems are almost the same when the amount of nano seed is larger than $3 \mathrm{wt} \%$. In the micro-synthesis system (e.g., $D_{0}=14.2$ $\mu \mathrm{m})$, when the seed size is smaller than $300 \mathrm{~nm}$, the equation can be simplified to $D=d x^{-1 / 3}$. Furthermore, the performance of B-ZSM-5 was related to its size in the catalytic conversion of methanol to propylene. With decreasing zeolite particle size, carbon formation is slowed and carbon capacity is increased. Adding only $1 \mathrm{wt} \%$ of $74 \mathrm{~nm}$ seed to the synthesis system can improve the practical catalyst life time from $46 \mathrm{~h}$ to $794 \mathrm{~h}$. 


\section{Acknowledgements}

The authors thank the financial support from the State Key Laboratory of Fine Chemicals (KF1605), the State Key Program of National Natural Science Foundation of China (21236008) and the QianRen Type B Award to CS from Chinese government.

\section{Notes and references}

1 A. Corma, Chem. Rev., 1995, 95, 559-614.

2 C. Dai, A. Zhang, M. Liu, X. Guo and C. Song, Adv. Funct. Mater., 2015, 25, 7479-7487.

3 C. dai, A. Zhang, M. Liu, L. Gu, X. Guo and C. Song, ACS Nano, 2016, 10, 7401-7408.

4 Y. Zhang, M. Xue, Y. Zhou, H. Zhang, W. Wang, Q. Wang and X. Sheng, RSC Adv., 2016, 6, 29410-29422.

5 Y. Zhao, H. Wu, W. Tan, M. Zhang, M. Liu, C. Song, X. Wang and X. Guo, Catal. Today, 2010, 156, 69-73.

6 C. Hammond, N. Dimitratos, R. L. Jenkins, J. A. LopezSanchez, S. A. Kondrat, M. Hasbi ab Rahim, M. M. Forde, A. Thetford, S. H. Taylor, H. Hagen, E. E. Stangland, J. H. Kang, J. M. Moulijn, D. J. Willock and G. J. Hutchings, ACS Catal., 2013, 3, 689-699.

7 C. Xu, H. Liu, M. Jia, J. Guan, S. Wu, T. Wu and Q. Kan, Appl. Surf. Sci., 2011, 257, 2448-2454.

8 K. Zhou, X. Hu, B. Chen, C. Hsueh, Q. Zhang, J. Wang, Y. Lin and C. Chang, Appl. Surf. Sci., 2016, 383, 300-309.

9 T. Zhang, J. Shi, J. Liu, D. Wang, Z. Zhao, K. Cheng and J. Li, Appl. Surf. Sci., 2016, 375, 186-195.

10 X. Lou, P. Liu, J. Li, Z. Li and K. He, Appl. Surf. Sci., 2014, 307, 382-387.

11 Z. Li, X. Chen, J. Li, X. Ren, S. Liu, J. Gao, J. W. Schwank, T. Zhang, W. Su and H. Chang, RSC Adv., 2016, 6, 102570102581.

12 B. Li, B. Sun, X. Qian, W. Li, Z. Wu, Z. Sun, M. Qiao, M. Duke and D. Zhao, J. Am. Chem. Soc., 2013, 135, 1181-1184.

13 C. Sun, J. Du, J. Liu, Y. Yang, N. Ren, W. Shen, H. Xu and Y. Tang, Chem. Commun., 2010, 46, 2671-2673.

14 Y. Yang, C. Sun, J. Du, Y. Yue, W. Hua, C. Zhang, W. Shen and H. Xu, Catal. Commun., 2012, 24, 44-47.

15 J. Liu, C. Zhang, Z. Shen, W. Hua, Y. Tang, W. Shen, Y. Yue and H. Xu, Catal. Commun., 2009, 10, 1506-1509.

16 D. Mores, J. Kornatowski, U. Olsbye and B. M. Weckhuysen, Chem.-Eur. J., 2011, 17, 2874-2884.

17 Y. Gao, B. Zheng, G. Wu, F. Ma and C. Liu, $R S C A d v .$, 2016, 6, 83581-83588.

18 A. Corma, Chem. Rev., 1997, 97, 2373-2419.

19 F. L. Bleken, K. Barbera, F. Bonino, U. Olsbye, K. P. Lillerud, S. Bordiga, P. Beato, T. V. W. Janssens and S. Svelle, J. Catal., 2013, 307, 62-73.

20 M. Milina, S. Mitchell, P. Crivelli, D. Cooke and J. PérezRamírez, Nat. Commun., 2014, 5, 3922.

21 Q. Zhang, S. Hu, L. Zhang, Z. Wu, Y. Gong and T. Dou, Green Chem., 2014, 16, 77-81.

22 X. Meng, F. Nawaz and F. Xiao, Nano Today, 2009, 4, 292301.
23 C. H. Christensen, I. Schmidt, A. Carlsson, K. Johannsen and K. Herbst, J. Am. Chem. Soc., 2005, 127, 8098-8102.

24 F. Liu, T. Willhammar, L. Wang, L. Zhu, Q. Sun, X. Meng, W. Carrillo-Cabrera, X. Zou and F. S. Xiao, J. Am. Chem. Soc., 2012, 134, 4557-4560.

25 C. Li, Y. Ren, J. Gou, B. Liu and H. Xi, Appl. Surf. Sci., 2017, 392, 785-794.

26 F. Meng, Y. Wang and S. Wang, RSC Adv., 2016, 6, 5858658593.

27 L. R. Aramburo, S. Teketel, S. Svelle, S. R. Bare, B. Arstad, H. W. Zandbergen, U. Olsbye, F. M. F. de Groot and B. M. Weckhuysen, J. Catal., 2013, 307, 185-193.

28 M. Firoozi, M. Baghalha and M. Asadi, Catal. Commun., 2009, 10, 1582-1585.

29 Y. Wang, C. Dai, B. Chen, Y. Wang, C. Shi and X. Guo, Catal. Today, 2015, 258, 616-626.

30 M. Khatamian, A. A. Khandar, M. Haghighi and M. Ghadiri, Appl. Surf. Sci., 2011, 258, 865-872.

31 H. Li, Y. Wang, F. Meng, H. Chen, C. Sun and S. Wang, RSC Adv., 2016, 6, 99129-99138.

32 G. Xiong, J. Yin, J. Liu, X. Liu, Z. Guo and L. Liu, RSC Adv., 2016, 6, 101365-101371.

33 V. Valtchev and L. Tosheva, Chem. Rev., 2013, 113, 67346760.

34 L. Chen, X. Li, J. C. Rooke, Y. Zhang, X. Yang, Y. Tang, F. Xiao and B. Su, J. Mater. Chem., 2012, 22, 17381-17403.

35 Z. Qin, L. Lakiss, L. Tosheva, J. Gilson, A. Vicente, C. Fernandez and V. Valtchev, Adv. Funct. Mater., 2014, 24, 257-264.

36 T. Xue, Y. M. Wang and M. He, Microporous Mesoporous Mater., 2012, 156, 29-35.

37 J. Dedecek, V. Balgová, V. Pashkova, P. Klein and B. Wichterlová, Chem. Mater., 2012, 24, 3231-3239.

38 S. Sang, F. Chang, Z. Liu, C. He, Y. He and L. Xu, Catal. Today, 2004, 93-95, 729-734.

39 H. Zhang, Y. Ma, K. Song, Y. Zhang and Y. Tang, J. Catal., 2013, 302, 115-125.

40 N. Ren, Z. Yang, X. Lv, J. Shi, Y. Zhang and Y. Tang, Microporous Mesoporous Mater., 2010, 131, 103-114.

41 N. Ren, J. Bronic, T. A. Jelic, A. Palcic and B. Subotic, Cryst. Growth Des., 2012, 12, 1736-1745.

42 N. Ren, J. Bronic, B. Subotic, X. Lv, Z. Yang and Y. Tang, Microporous Mesoporous Mater., 2011, 139, 197-206.

43 N. Ren, J. Bronic, B. Subotic, Y. Song, X. Lv and Y. Tang, Microporous Mesoporous Mater., 2012, 147, 229-241.

44 G. Majano, A. Darwiche, S. Mintova and V. Valtchev, Ind. Eng. Chem. Res., 2009, 48, 7084-7091.

45 J. C. Jansen, Stud. Surf. Sci. Catal., 1991, 58, 77-136.

46 Z. Gabelica, E. G. Derouane and N. Blom, Adv. Chem. Ser., 1984, 248, 219-251.

47 E. G. Derouane and Z. Gabelica, J. Solid State Chem., 1986, 64, 296-304.

48 M. Ghamami and L. B. Sand, Zeolites, 1983, 3, 155-162.

49 F. Crea, A. Nastro, J. B. Nagy and R. Aiello, Zeolites, 1988, 8, 262-267. 